\title{
Efecto de la Musicoterapia sobre los Niveles de Estrés de los Usuarios Internos de la Clínica de Odontología de la Universidad del Desarrollo
}

\author{
Music Therapy Effect on Stress Levels of Internal Users \\ of the Dental clinic of universidad del desarrollo
}

Loreto Bancalari S. \& Patricio Oliva M.

BANCARALI, S. L. \& OLIVA, M. P. Efecto de la musicoterapia sobre los niveles de estrés de los usuarios internos de la clínica de odontología de la universidad del desarrollo. Int. J. Odontostomat., 6(2):189-193, 2012.

RESUMEN: La musicoterapia consiste en una psicoterapia que involucra al sonido y todos sus componentes, con el fin de conseguir distintos estados terapéuticos a nivel psicológico y psicomotriz del paciente, dentro de los cuales cabe destacar la reducción en los niveles de estrés. El objetivo fue determinar el efecto de la musicoterapia aplicada durante la sesión clínica, sobre los niveles de estrés de los usuarios internos (alumnos, tutores y asistentes dentales) de la Clínica de Odontología de la Universidad del Desarrollo. Se realizó un estudio de intervención con una población de 84 individuos agrupados previamente en 3 grupos correspondientes a alumnos, tutores y asistentes dentales; cada uno de los cuales se dividió en grupo intervenido (sometido a la aplicación de musicoterapia) y un grupo control, al cual no se le aplicó nada. La musicoterapia implementada, consistió en la aplicación de música de relajación durante una semana de clínicas. Mediante una encuesta ad hoc para la investigación se procedió a recolectar los datos de estrés inicial y final a esa semana, tanto para el grupo experimental como para del control. Se realizó análisis estadístico mediante el programa SPSS 17@ la normalidad evaluada mediante ShapiroWilK, arrojó resultados no paramétricos, se aplicó la prueba de Mann-Withney para los 3 grupos analizados. Los datos obtenidos muestran que el cambio en el nivel de estrés entre el grupo control y el grupo experimental en los alumnos, tutores y asistentes dentales, arrojando valores p menores a 0,05 $(0,000,0,015,0,026$ respectivamente), demostrando que la musicoterapia disminuye los niveles de estrés de los usuarios internos. La musicoterapia, aplicada durante la sesión dental, disminuye los niveles de estrés de los individuos.

PALABRAS CLAVE: musicoterapia, estrés, usuarios internos.

\section{INTRODUCCION}

El estrés es considerado como una reacción psicofisiológica del organismo como respuesta adaptativa ante situaciones complejas. Esta manifestación es de importancia médica, ya que además conlleva consecuencias tales como irritabilidad, estados depresivos, ansiedad y potencial factor de riesgo para contraer enfermedades crónicas e infectocontagiosas (Sanchez, 2006), siendo además, una causa importante de ausencias y bajo rendimiento en el trabajo (García-Viniegras et al., 1997).

En odontología se estima que entre el 10 y el $15 \%$ del total de la población sufre de algún trastorno de ansiedad frente al tratamiento odontológico (Lima Álvarez \& Casanova Rivero, 2006) ya que es percibida como una experiencia agónica (Martino, 1987). Se han implementado diversas terapias que ayudan a disminuir los niveles de estrés de los pacientes, que van desde lo no invasivo hasta métodos como la farmacoterapia o la sedación. Sin embargo, todos estos están orientados hacia el paciente, excluyendo a los dentistas y sus asistentes, quienes por la naturaleza de su trabajo, necesitan de procedimientos que no implique sedación para la disminución del estrés, como la musicoterapia.

La musicoterapia es un tratamiento sistemático de la música, con el objeto de lograr cambios de conducta (Suzanne, 1987), induciendo estados de relajación a nivel cerebral (Campbell, 1997) influyendo en 
la memoria y capacidad intelectual (Zarate \& Díaz, 2001) disminuyendo la ansiedad y el stress (Mitchell \& MacDonald, 2006) y aumentando la motivación y control (Aigen, 1999). Es considerado como un método simple, no invasivo, económico y con buena acogida por los pacientes (Suzanne). La utilización de la musicoterapia aumenta la motivación, eleva el humor y refuerza los sentimientos de responsabilidad (AlonsoCardaño et al., 2008) corroborando mediante la evidencia los efectos beneficiosos de la implementación de este tipo de estrategias para el control o disminución del estrés y sus efectos.

Actualmente, es un procedimiento aceptado por muchos centros de salud en países desarrollados, sin embargo, en Chile el desarrollo de esta disciplina es todavía bastante incipiente.

En respuesta a esa necesidad, el estudio presente determina el efecto que produce la musicoterapia aplicada durante la sesión clínico-dental sobre los niveles de estrés de alumnos, tutores y asistentes dentales.

\section{MATERIAL Y MÉTODO}

Se diseño una investigación de intervención longitudinal, randomizándo a la población en dos grupos, un grupo intervenido sometido a la aplicación de musicoterapia y otro grupo de control al cual no se le aplicó la terapia. La intervención duro una semana, realizándose mediciones del nivel de estrés antes de comenzar la semana y después de finalizada.

La población de estudio correspondió a todos los usuarios internos de la Clínica de Odontología de 5to año de la UDD-Concepción durante el 2011, implica a alumnos, tutores y asistentes dentales de las clínicas de adulto de la Facultad de Odontología de la Universidad del Desarrollo, Concepción.

El universo fue de 84 individuos, dentro de los cuales se encontraron 64 alumnos ( 36 grupo intervención, 28 grupo control), 14 tutores (8 grupo intervención, 6 grupo control) y 6 asistentes dentales ( 3 grupo intervención, 3 grupo control). Todos ellos se encontraban previamente agrupados en 4 grupos de clínica.

Los criterios de inclusión fueron: alumnos de 5 to año de pregrado que estuviesen cursando con la asignatura de Rehabilitación Oral de Adultos II, a los tuto- res y las asistentes de las clínicas de adultos de 5to año. Los criterios de exclusión implicó: tutores o asistentes de reemplazo y a los alumnos en recuperación de clínica, que hayan congelado el año o se hayan retirado de la carrera.

Para la selección de los grupos intervención y control se realizó una randomización por bloques de los grupos. Se recolectaron los datos mediante el uso de una escala adaptada para la investigación, las cuales fueron desarrolladas en base a una modificación de la Escala de Maslach para estrés laboral (Maslach \& Jackson, 1986) en donde se realizaron 18 preguntas que abordasen las manifestaciones psicológicas del participante con el fin de determinar su nivel de estrés.

Estos instrumentos de recolección de datos, fueron validados mediante juicio de expertos, entre los que se encontraron 2 cirujanos dentistas y 3 investigadores, evaluándose mediante el alfa de Cronbach. Además se realizó una prueba piloto con 5 personas, para evaluar la capacidad de comprensión de ellas. A cada participante se le aplicó la misma encuesta antes de comenzar la semana de estudio y la otra al finalizar ésta. La primera encuesta la cual tenía por objetivo medir el nivel de estrés basal de los participantes, mientras que la segunda encuesta aplicada al finalizar la semana, tenía por objetivo registrar el nivel de estrés final de los participantes.

Durante la semana de estudio, para el grupo control no hubo cambios y sus jornadas clínicas se realizaron de manera normal. En cambio, para el grupo intervención se aplicó la música mediante el uso del equipo de audio conectado con 2 parlantes ubicados estratégicamente en la clínica, a un volumen moderado, de manera que todos los participantes pudiesen escucharla con claridad. Esta intervención se realizó en las 4 sesiones clínicas de una semana normal, y fue aplicada durante toda la sesión (3 horas). El equipo fue instalado antes de comenzar cada sesión clínica correspondiente y retirada finalizada ésta, por el personal de la universidad.

La música que se utilizó a aquellas con efecto relajante y canciones contemporáneas elegidas por los mismos participantes mediante una recopilación previa de las canciones que a ellos les gustase escuchar. Este repertorio musical fue sometido a una prueba piloto con 5 individuos, para certificar su efecto relajante. Además, se debe señalar que toda la música fue configurada con el programa Power Sound Editor 
7.5.1 de manera que todas las canciones se escuchasen a un mismo volumen. Los datos obtenidos fueron tabulados en Microsoft Excel 2007® y luego exportados a SPSS 17@ para su posterior análisis.

Para demostrar la distribución de los datos se utilizó diagrama de cajas. La normalidad de los datos fue evaluada mediante la prueba de Shapiro-Wilks. La diferencia de medias entre grupos fue evaluada mediante la prueba no paramétrica de Mann-Whitney. Se trabajó con una confiabilidad de un $95 \%$, un $5 \%$ de significancia y una heterogeneidad del $50 \%$.

Los aspectos éticos consideró el consentimiento informado de acuerdo con las normas éticas de la Declaración de Helsinki de 1975, enmendada en el año 2005 que fue firmado por los sujetos. A cada participante se le informó sobre el estudio, explicando detalladamente. Una copia de este documento fue entregado al participante y la otra quedó guardada en los archivos de la investigación.

\section{RESULTADOS}

Se evaluó el cambio entre estrés inicial y final de una semana en alumnos, asistentes y tutores que se encuentran en las clínicas de la facultad, tras la aplicación de musicoterapia para el grupo intervenido. El proceso de encuestaje también se aplicó al gru-

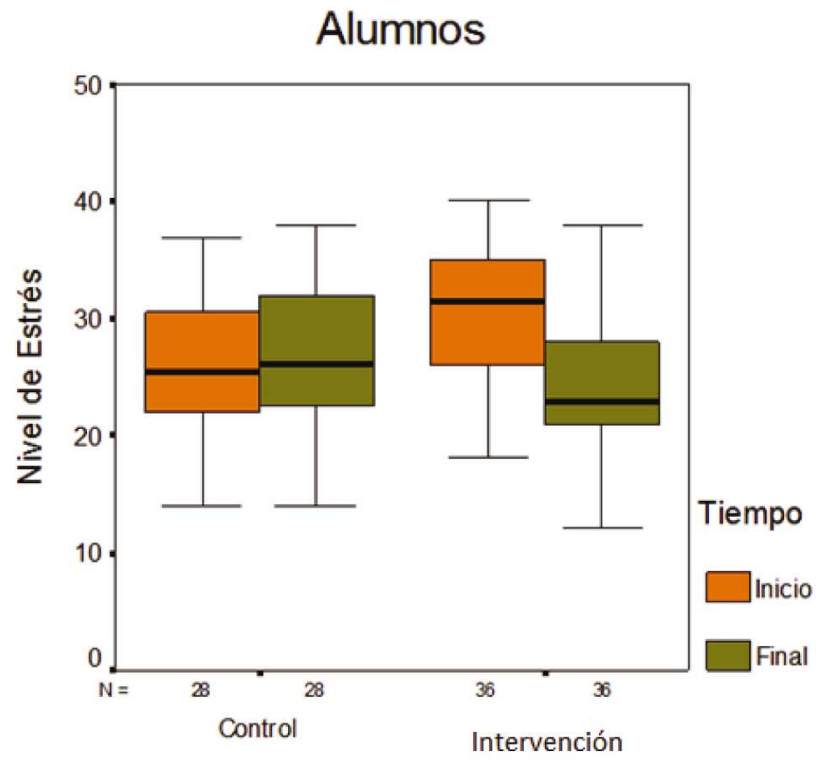

Grupo

Fig. 1. Variación en el nivel de estrés de Alumnos. po de control, pero sin la intervención de la musicoterapia.

En los alumnos (Fig. 1) en el grupo de control las medianas (como los brazos) no presentaron variacioones evidentes en el grupo control entre las dos mediciones. En el grupo intervención existió una modificación hacia la baja (disminución de estrés) entre la primera evaluación y la última, una vez utilizada la musicoterapia.

En los tutores clínicos (Fig. 2) el grupo de control (sin la musicoterapia) no tuvo variación entre la medición inicial y final de estrés. En el grupo de intervención (con musicoterapia) si existió una variación hacia la baja en el trascurso del tiempo, por lo cual existió una disminución del estrés.

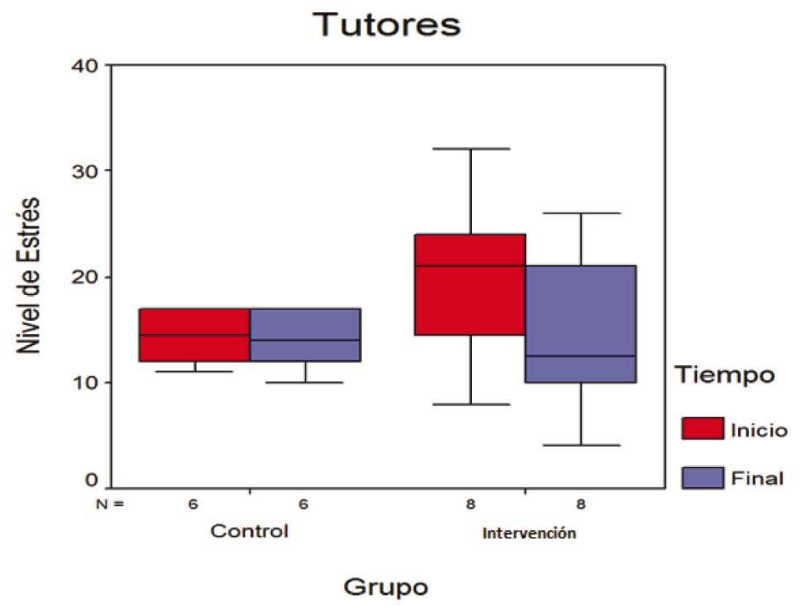

Fig. 2. Variación en el nivel de estrés de tutores.

En asistentes dentales (Fig. 3) el grupo de control (sin la musicoterapia) no presentó variación de acuerdo a las mediciones efectuadas. En el grupo de intervención (con musicoterapia) existió una variación evidente entre la primera medición y la última (visible en la caja y la mediana de ambas mediciones) implicando una disminución del estrés.

Para los datos tabulados se analizó la normalidad de los datos con la prueba de Shapiro-Wilk, lo cual arrojó distribuciones no paramétricas para todos los grupos, por lo cual se decidió aplicar la prueba no paramétrica de Mann-Whitney, debido a que las observaciones de los grupos son independientes, con variables ordinales o continuas y distribuciones no son paramétricas. 


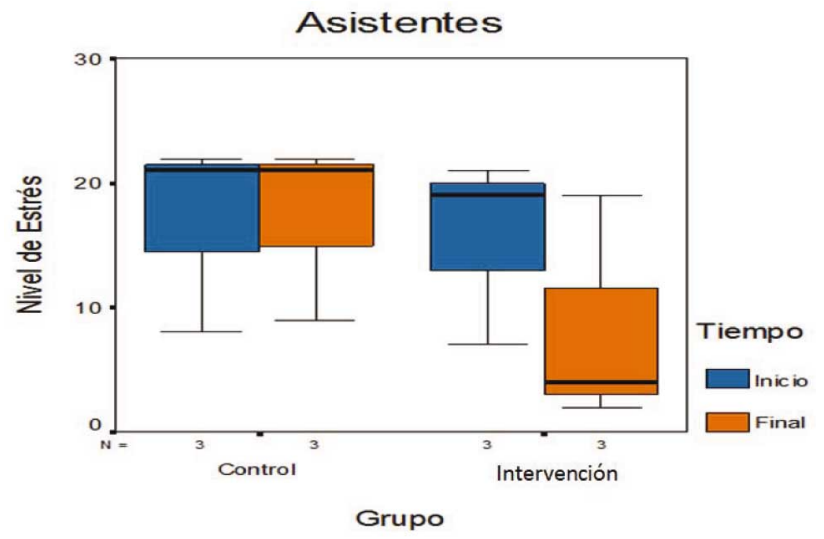

Fig. 3. Variación en el nivel de estrés de asistentes dentales.

La variación en el nivel de estrés entre el grupo control y el grupo intervención en los alumnos, tutores y asistentes dentales es estadísticamente significativo, arrojando valores $p$ menores a $0,05(0,000,0,015$ y 0,026 respectivamente), demostrando que la musicoterapia disminuye los niveles de estrés de los usuarios internos.

La prueba de Mann-Whitney arrojo una significatividad de 0,00 , menor a 0,05 , por lo cual existió una diferencia significativa entre las mediciones de estrés mediante la escala de Maslach en los alumnos que se encuentran en la clínica odontológica de la facultad.

La prueba de Mann-Whitney arrojó una significatividad de 0,015 , menor a 0,05 , por lo cual existió una diferencia significativa entre las mediciones de estrés mediante la escala de Maslach en los tutores que se encuentran en la clínica odontológica.

La prueba de Mann-Whitney arrojó una significatividad de 0,026, menor a 0,05, por lo cual existió una diferencia significativa entre las mediciones de estrés mediante la escala de Maslach en los asistentes que se encuentran en la clínica odontológica de la facultad.

\section{DISCUSIÓN}

Considerando que no existen estudios que avalen el efecto de la musicoterapia en funcionarios clínicos (solo en pacientes), los hallazgos del estudio poseen una relevancia clínica, debido a que demuestran que existe una diferencia significativa en los niveles de estrés posterior a la aplicación de la musicoterapia entre el grupo control e intervención de los usuarios internos en una clínica odontológica tipo.

La metodología para la investigación apuntaba al control de confusores, sobre todo al poseer un grupo de control en el cual se reproduce el contexto clínico odontológico típico. En el grupo control no se observaron diferencias significativas en relación al nivel de estrés inicial con el final (no hubo intervención que pudiera influir en el estrés cotidiano), no ocurre lo mismo en el grupo intervenido, que presenta variaciones en el nivel de estrés, disminuyendo de acuerdo a la percepción de los usuarios internos expresadas en la escala de medición, coincidiendo con los resultados de otros estudios, también en contextos clínicos odontológicos, pero en usuarios externos (Lima Álvarez \& Casanova Rivero, 2006)

De acuerdo a los resultados se concluye que la musicoterapia, aplicada durante la sesión clínica, es efectiva para disminuir el nivel de estrés de un individuo. El estrés causado por los deberes y quehaceres del trabajo clínico, es mitigado por medio de este tipo de terapias.

BANCARALI, S. L. \& OLIVA, M. P. Music therapy effect on stress levels of internal users of the dental clinic of universidad del desarrollo. Int. J. Odontostomat., 6(2):189-193, 2012.

SUMMARY: Music therapy is a psychotherapy that involves sound and all its components, in order to get different therapeutic states in psychological and psychomotor level of the patient, within which included the reduction in stress levels. The aim was to determine the effect of music therapy applied during the clinical session, concerning stress levels of internal users (students, tutors and dental assistants) of the Dental Clinic of the Universidad del Desarrollo. An intervention study with a population of 84 individuals, previously grouped into 3 groups for students, tutors and dental assistants, each of which was divided into intervention group (subject to the application of music therapy) and a group control, to which nothing was applied to them. Implemented the music therapy consisted of the application of relaxation music for a week of clinics. Through an ad hoc survey research, we proceeded to collect data from initial and final stress for that week, for the experimental and control groups. Statistical analysis was performed using SPSS 17 @ ShapiroWilK assessed by normal, non-parametric results showed, was applied the Mann-Whitney test for the 3 groups analyzed. The data obtained show that the change in stress levels between control and experimental group in students, tutors and dental assistants, shows $P$ values less than 0.05 ( $0.000,0.015,0.026$ respectively), demonstrating that Music therapy reduces stress levels of internal users. Music therapy applied during the session dental, decreases stress levels of individuals.

KEY WORDS: music therapy, stress, internal users. 


\section{REFERENCIAS BIBLIOGRÁFICAS}

Alonso-Cardaño, A.; Hernaez-Martínez, M. \& MartíAuge, P. Tratamiento multidisciplinar para el dolor lumbar crónico: Programa de musicoterapia. Rev. Soc. Esp. Dolor, 15(4):228-33, 2008.

Aigen, $\mathrm{K}$. The true nature of music-centered music therapy fheory. Br. J. Mus. Ther., 13(2):77-82, 1999.

Campbell, D. El efecto Mozart, El sonido y la curación. Barcelona, España, Edición Urano, 1997. pp.71-8.

García-Viniegras, C.; Rodríguez, M. J.; Barbón, R. D. \& Cárdenas, E. N. Musicoterapia: Una modalidad terapéutica para el estrés laboral. Rev. Cubana Med. Gen. Integr., 13(6):538-43, 1997.

Lima Álvarez, M. \& Casanova Rivero, Y. Miedo, ansiedad y fobia al tratamiento estomatológico. Rev. Hum. Med., 6(1):0-0, 2006. Disponible en: http:// scielo.s/d.cu/scielo.php?script=sci_arttext \&pid=S1727-81202006000100007\&/ng=es

Martino, B. K. Ansiedad. Una revisión bibliográfica. Clin. Odontol. Nort. Amer., 31(1):15-22, 1987.

Maslach, C. \& Jackson, S. E. Maslach Burnout Inventory. Palo Alto, Consulting Psychlogists Press, 1986.

Mitchell, L. A. \& MacDonald, R. A. An experimental investigation of the effects of preferred and relaxing music listening on pain perception. J. Music Ther., 43(4):295-316, 2006.

Sánchez, M.; González, R.; Marsan, V. \& Macías, C, Asociación entre el estrés y las enfermedades infecciosas, autoinmunes, neoplásicas y cardiovasculares. Rev. Cubana Hematol. Inmunol. Med. Transf., 22(3), 2006. Disponible en: http:// bvs.sld.cu/revistas/hih/vol22_3_06/hih02306.html

Suzanne, B. Music therapist's handbook. St. Louis, Warren H. Green, Inc., 1987. pp.3-15.

Zárate, D. P. \& Díaz, T. V. Aplicaciones de la musicoterapia en la medicina. Rev. Méd. Chile, 129(2): 219-23, 2001.
Dirección de correspondencia:

Loreto Bancalari Simon

Facultad de Odontología

Universidad del Desarrollo

Barros Arana 1734, Concepción

CHILE

Fono: 041-2268544

Fax: 56-41-2268501

Email: loretobancalaris@gmail.com

Recibido : 20-04-2012

Aceptado: 28-05-2012 